

Algebraic Structures and Their Applications Vol. 4 No. 2 ( 2017 ) pp 39-43.

\title{
NOTE ON REGULAR AND COREGULAR SEQUENCES
}

\author{
H. SAREMI*
}

Communicated by S. Alikhani

\begin{abstract}
Let $R$ be a commutative Noetherian ring and let $M$ be a finitely generated $R$ module. If $I$ is an ideal of $R$ generated by $M$-regular sequence, then we study the vanishing of the first Tor functors. Moreover, for Artinian modules and coregular sequences we examine the vanishing of the first Ext functors.
\end{abstract}

\section{INTRODUCTION}

Throughout this note, we shall assume that $R$ is a commutative Noetherian ring with nonzero identity and I an ideal of $R$. Suppose $M$ and $A$ two non-zero finitely generated and Artinian $R$-modules, respectively. An ordered sequence $x_{1}, \ldots, x_{t}$ of elements of $R$ is called an $M$-regular sequence [山, Definition 1.1.1]; if $x_{i}$ is not a zero-divisor in $M /\left(x_{1}, \ldots, x_{i-1}\right) M$ for $i=1, \ldots, t$, and $M \neq\left(x_{1}, \ldots, x_{t}\right) M$. Also, an ordered sequence $x_{1}, \ldots, x_{t}$ of elements of $R$ is said an $A$-coregular sequence [3, Definition 3.1]; if $x_{i}$ is an $\left(0:_{A}\left(x_{1}, \ldots, x_{i-1}\right)\right)$-coregular element for $i=1, \ldots, t$, and $\left(0:_{A}\left(x_{1}, \ldots, x_{t}\right)\right) \neq 0$. Note that an element $x$ of $R$ is $A$-coregular if $A=x A$, (see [3, Definition 2.4]).

DOI:http://dx.doi.org/10.29252/asta.4.2.39

MSC(2010): Primary: 13D15. Secondary: 13D07.

Keywords: regular sequence, coregular sequence, ring.

Received: 14 September 2017, Accepted: 18 September 2018

*Corresponding author

(C) 2017 Yazd University. 
It is known that if $I$ is generated by an $M$-regular sequence, then $\operatorname{Tor}_{1}^{R}(R / I, M)=0$. In particular, if $x_{1}, \ldots, x_{t}$ is an R-regular sequence, $I=\left(x_{1}, \ldots, x_{s}\right)$ and $J=\left(x_{s+1}, \ldots, x_{t}\right)$ two ideals of $R$ then $\operatorname{Tor}_{1}^{R}(R / I, R / J)=0$ and so $I \cap J=I J$.

Our main aim in this article is to extend Proposition 1.1.4 of [⿴囗十 for powers an ideal that generated by regular sequence and use it over Tor functors. Additionally, we examine the problem on the self-duality.

\section{The RESUlts}

The interplay between regular sequences and homological invariants is a major theme, and numerous arguments will be based on the following proposition which is appear in [प]].

Proposition 2.1. Let $R$ be a ring, $M$ an $R$-module, and $x$ an $M$-regular sequence. Then an exact sequence

$$
N_{2} \stackrel{f_{2}}{\longrightarrow} N_{1} \stackrel{f_{1}}{\longrightarrow} N_{0} \stackrel{f_{0}}{\longrightarrow} M \longrightarrow 0
$$

induces an exact sequence

$$
\frac{N_{2}}{x N_{2}} \longrightarrow \frac{N_{1}}{x N_{1}} \longrightarrow \frac{N_{0}}{x N_{0}} \longrightarrow \frac{M}{x M} \longrightarrow 0
$$

The following result is a generalization of Proposition 2.1.

Theorem 2.2. Let $(R, \mathfrak{m})$ be a local ring, $M$ a finitely generated $R$-module, and $I=\left(a_{1}, \ldots, a_{t}\right)$ an ideal generated by an $M$-regular sequence. Then an exact sequence

$$
N_{2} \stackrel{f_{2}}{\longrightarrow} N_{1} \stackrel{f_{1}}{\longrightarrow} N_{0} \stackrel{f_{0}}{\longrightarrow} M \longrightarrow 0,
$$

of $R$-modules induces an exact sequence

$$
\frac{N_{2}}{I^{n} N_{2}} \stackrel{\overline{f_{2}}}{\longrightarrow} \frac{N_{1}}{I^{n} N_{1}} \stackrel{\overline{f_{1}}}{\longrightarrow} \frac{N_{0}}{I^{n} N_{0}} \stackrel{\overline{f_{0}}}{\longrightarrow} \frac{M}{I^{n} M} \longrightarrow 0,
$$

for all $n \geq 1$.

Proof. Since tensor product is a right exact functor, we only need to verify exactness at $\frac{N_{1}}{I^{n} N_{1}}$. let ${ }^{-}$denote residue classes modulo $I^{n} N_{1}$. Let $y_{1} \in N_{1}$ such that $\overline{f_{1}}(\bar{y})=0$. Then $f_{1}(y) \in$ $I^{n} N_{0}$. Hence we can consider a homogeneous polynomial of degree $n, f_{1}(y) \in N_{0}\left[x_{1}, \ldots, x_{t}\right]$ such that

$$
f_{1}(y)=\sum_{\alpha_{i_{1}}+\ldots+\alpha_{i_{t}}=n} \alpha_{i_{1} \ldots i_{t}} x_{1}^{\alpha_{i_{1}}} \ldots x_{t}^{\alpha_{i_{t}}}
$$

for some $\alpha_{i_{1} \ldots i_{t}} \in N_{0}$. Since

$$
\sum_{\alpha_{i_{1}}+\ldots+\alpha_{i_{t}}=n} f_{0}\left(\alpha_{i_{1} \ldots i_{t}}\right) x_{1}^{\alpha_{i_{1}}} \ldots x_{t}^{\alpha_{i_{t}}}=f_{0} \circ f_{1}(y)=0 \in I^{n+1} M
$$

by [ [U, Theorem 1.1.7] or [2], Theorem 16.2] the coefficients $f_{0}\left(\alpha_{i_{1} \ldots i_{t}}\right) \in I M$, and so $f_{0}\left(\alpha_{i_{1} \ldots i_{t}}\right)=\sum_{j=1}^{t} m_{i_{j}} x_{j}$ for some $m_{j_{i}} \in M$. By replacing $m_{j_{i}}$ in the equation $(* *)$ and 
repeating this argument, we have $f_{0}\left(\alpha_{i_{1} \ldots i_{t}}\right) \in I^{n} M$ for all $n \in \mathbb{N}$. By Krull intersection Theorem, $f_{0}\left(\alpha_{i_{1} \ldots i_{t}}\right)=0$ and by our hypothesis $\alpha_{i_{1} \ldots i_{t}} \in \operatorname{im} f_{1}$. Hence there exists $\beta_{i_{1} \ldots i_{t}} \in N_{1}$ such that $\alpha_{i_{1} \ldots i_{t}}=f_{1}\left(\beta_{i_{1} \ldots i_{t}}\right)$. Therefore by replacing this value in the equation $(*)$ and our hypothesis, there exists $z \in N_{2}$ such that $y-\sum_{\alpha_{i_{1}}+\ldots+\alpha_{i_{t}}=n} \beta_{i_{1} \ldots i_{t}} x_{1}^{\alpha_{i_{1}}} \ldots x_{t}^{\alpha_{i_{t}}}=f_{2}(z)$. Hence $\bar{y}=\overline{f_{2}}(\bar{z})$, as desired.

Corollary 2.3. Let $(R, \mathfrak{m})$ be a local ring, $M$ a finitely generated $R$-module and $x_{1}, \ldots, x_{s}, x_{s+1}, \ldots, x_{t}$ an $M$-regular sequence. Then the following statements hold:

(i) If $I=\left(x_{1}, \ldots, x_{s}\right)$, then $\operatorname{Tor}_{1}^{R}\left(R / I^{n}, M\right)=0$ for all $n \geq 1$.

(ii) If $J=\left(x_{s+1}, \ldots, x_{t}\right)$, then $\operatorname{Tor}_{1}^{R}\left(R / J^{m}, M / I^{n} M\right)=0$ for all $m, n \geq 1$.

Proof. (i) From the exact sequence $0 \longrightarrow K \longrightarrow F \longrightarrow M \longrightarrow 0$, where $F$ is a free $R$-module and by using Theorem 2.1, we obtain the result.

(ii) It is known that $\operatorname{Ass}\left(M / I^{n} M\right)=\operatorname{Ass}(M / I M)$. It therefore follows

$\operatorname{Ass}\left(M / I^{n} M+\left(x_{s+1}, \ldots, x_{i}\right) M\right)=\operatorname{Ass}\left(M / I M+\left(x_{s+1}, \ldots, x_{i}\right) M\right)$ for all $n \in \mathbb{N}$ and $s+1 \leq i \leq$ $t$. Hence $x_{s+1}, \ldots, x_{t}$ is an $M / I^{n} M$-regular sequence for all $n \in \mathbb{N}$ and so by Theorem 2.2 , the result follows.

Corollary 2.4. Let $(R, \mathfrak{m})$ be a local ring and $J \subseteq I$ be two ideals of $R$ such that $I / J$ is generated by $R / J$-regular sequence. Then $I^{n} \cap J=I^{n-1} J$ for all $n \in \mathbb{N}$.

Proof. From the exact sequence

$$
0 \longrightarrow \mathfrak{m} I+J / \mathfrak{m} I \longrightarrow I / \mathfrak{m} I \longrightarrow I / \mathfrak{m} I+J \longrightarrow 0
$$

and by using Nakayama's Lemma, There exists $x_{1}, \ldots, x_{s}, y_{1}, \ldots, y_{t}$ of $R$ such that $I=$ $\left(x_{1}, \ldots, x_{s}, y_{1}, \ldots, y_{t}\right), J=\left(x_{1}, \ldots, x_{s}\right)$ and $I / J=\left(\overline{y_{1}}, \ldots, \overline{y_{t}}\right)$, where $y_{1}, \ldots, y_{t}$ is an $R / J$ regular sequence. By Corollary 2.3(i) $\operatorname{Tor}_{1}^{R}\left(R /\left(y_{1}, \ldots, y_{t}\right)^{n}, R / J\right)=0$ for all $n \in \mathbb{N}$ and so $J \cap\left(y_{1}, \ldots, y_{t}\right)^{n}=J\left(y_{1}, \ldots, y_{t}\right)^{n}$. Therefore $J \cap I^{n}=J \cap\left(J^{n}+J^{n-1}\left(y_{1}, \ldots, y_{t}\right)+\ldots+\right.$ $\left.J\left(y_{1}, \ldots, y_{t}\right)^{n-1}+\left(y_{1}, \ldots, y_{t}\right)^{n}\right)=J^{n}+J^{n-1}\left(y_{1}, \ldots, y_{t}\right)+\ldots+J\left(y_{1}, \ldots, y_{t}\right)^{n-1}+J \cap\left(y_{1}, \ldots, y_{t}\right)^{n}=$ $J I^{n-1}$.

Proposition 2.5. Let $M$ be a finitely generated $R$-module and $I=\left(a_{1}, \ldots, a_{t}\right)$ an ideal generated by an $M$-regular sequence and an $R$-regular sequence. Then $\operatorname{Tor}_{i}^{R}\left(\frac{R}{I^{n}}, M\right)=0$ for all $n \geq 1$ and all $i \geq 1$. 
Proof. We use induction on $n$. If $n=1$, then $\operatorname{Tor}_{i}^{R}\left(\frac{R}{I}, M\right)=0$ for all $i \geq 1$, by Theorem 2.2 . let $n>1$ and suppose that the proposition holds for all integer smaller than $n$. By using the exact sequence

$$
0 \longrightarrow \frac{I^{n-1}}{I^{n}} \longrightarrow \frac{R}{I^{n}} \longrightarrow \frac{R}{I^{n-1}} \longrightarrow 0
$$

and the inductive hypothesis, $\operatorname{Tor}_{i}^{R}\left(\frac{R}{I^{n}}, M\right) \cong \operatorname{Tor}_{i}^{R}\left(\frac{I^{n-1}}{I^{n}}, M\right)$ for all $i \geq 1$. Hence, by us-

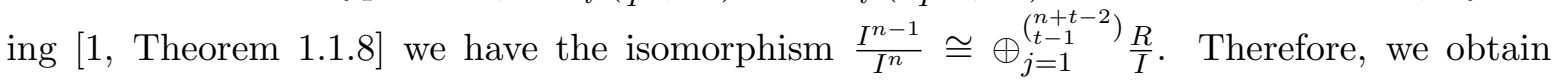
$\operatorname{Tor}_{i}^{R}\left(\frac{R}{I^{n}}, M\right)=0$ for all $n \geq 1$ and all $i \geq 1$. This completes the inductive step.

Corollary 2.6. Let $(R, \mathfrak{m})$ be a Cohen-Macaulay local ring, $M$ a finitely generated maximal Cohen-Macaulay $R$-module, and $I=\left(a_{1}, \ldots, a_{t}\right)$ an ideal generated by an $R$-regular sequence. Then $\operatorname{Tor}_{i}^{R}\left(\frac{R}{I^{n}}, M\right)=0$ for all $i \geq 1$ and all $n \geq 1$.

Proof. Since every $R$-regular sequence is an $M$-regular sequence, the result follows by Proposition 2.5.

The following result is a dual of [प, Proposition 1.1.4].

Proposition 2.7. Let $A$ be an Artinian $R$-module and $I=\left(a_{1}, \ldots, a_{t}\right)$ an ideal generated by an A-coregular sequence. Then an exact sequence

$$
0 \longrightarrow A \stackrel{g_{0}}{\longrightarrow} B_{0} \stackrel{g_{1}}{\longrightarrow} B_{1} \stackrel{g_{2}}{\longrightarrow} B_{2}
$$

of $R$-modules induces an exact sequence

$$
0 \longrightarrow\left(0::_{A} I\right) \stackrel{g_{0} \mid}{\longrightarrow}\left(0:_{B_{0}} I\right) \stackrel{g_{1} \mid}{\longrightarrow}\left(0:_{B_{1}} I\right) \stackrel{g_{2} \mid}{\longrightarrow}\left(0:_{B_{1}} I\right) .
$$

Proof. By induction, it is enough to consider the case in which $I$ consists of a single $A$-coregular element $a=a_{1}$. We obtain the induced sequence if we use the functor $\operatorname{Hom}_{R}\left(\frac{R}{I},-\right)$. Since $\operatorname{Hom}_{R}\left(\frac{R}{I},-\right)$ is a left exact functor, we only need to verify exactness at $\left(0:_{B_{1}} a\right)$. Let $g \mid$ is a reduced homomorphism of $g$. If $g_{2} \mid(\alpha)=g_{2}(\alpha)=0$ for some $\alpha \in\left(0:_{B_{1}} a\right)$, then $\alpha \in \operatorname{Ker} g_{2}=$ $\operatorname{im} g_{1}$ and hence there is $\beta \in B_{0}$ with $\alpha=g_{1}(\beta)$. It follows that $a \beta \in \operatorname{Ker} g_{1}=\operatorname{im} g_{0}$ and so there is $\gamma \in A$ with $a \beta=g_{0}(\gamma)$. Since $a$ is an $A$-coregular element, we have $A=a A$ (see [3], Definition 2.4]), and so there is $\gamma^{\prime} \in A$ with $\gamma=a \gamma^{\prime}$. It follows that $a\left(\beta-g_{0}\left(\gamma^{\prime}\right)\right)=0$. Hence $\beta-g_{0}\left(\gamma^{\prime}\right) \in\left(0:_{B_{0}} a\right)$, and $\alpha=g_{1}\left(\beta-g_{0}\left(\gamma^{\prime}\right)\right)=g_{1}(\beta) \in \operatorname{im} g_{1} \mid$, as desired.

Corollary 2.8. Let $A$ be an Artinian $R$-module and $I=\left(a_{1}, \ldots, a_{t}\right)$ an ideal generated by an $A$-coregular sequence. Then $\operatorname{Ext}_{R}^{1}\left(\frac{R}{I}, A\right)=0$. 
Alg. Struc. Appl. Vol. 4 No. 2 (2017) 39-43.

Proof. From the exact sequence $0 \longrightarrow A \longrightarrow E \longrightarrow K \longrightarrow 0$, where $E$ is an injective $R$-module and by using Proposition 2.7, we obtain the result.

Question 2.9. (with hypothesis as Proposition 2.7) Is the following

$$
0 \longrightarrow\left(0::_{A} I^{n}\right) \stackrel{g_{0} \mid}{\longrightarrow}\left(0:_{B_{0}} I^{n}\right) \stackrel{g_{1} \mid}{\longrightarrow}\left(0:_{B_{1}} I^{n}\right) \stackrel{g_{2} \mid}{\longrightarrow}\left(0:_{B_{1}} I^{n}\right),
$$

exact sequence for all $n \geq 1$ ?

Acknowledgement . I would like to thank deeply grateful to the referee for the careful reading of the manuscript and the helpful suggestions.

\section{REFERENCES}

[1] W. Bruns and J. Herzog, Cohen-Macaulay rings, Cambridge University Press, Cambridge, UK, (1998).

[2] H. Matsumura, Commutative ring theory, Cambridge University Press, Cambridge, UK, (1986).

[3] A. Ooishi, Matlis duality and width of a module, Hiroshima Math. J., 6 (1976), 573-587.

\section{Hero Saremi}

Department of Mathematics, Sanandaj Branch

Islamic Azad University

Sanandaj, Iran.

hero.saremi@gmail.com 